\section{BMJ Open Respiratory Research}

\title{
Association between influenza vaccination and hospitalisation or all- cause mortality in people with COVID-19: a retrospective cohort study
}

\author{
Christopher R Wilcox, ${ }^{1}$ Nazrul Islam, ${ }^{2}$ Hajira Dambha-Miller ${ }^{1}$
}

To cite: Wilcox CR, Islam N, Dambha-Miller H. Association between influenza vaccination and hospitalisation or all-cause mortality in people with COVID-19: a retrospective cohort study. BMJ Open Resp Res 2021;8:e000857. doi:10.1136/ bmjresp-2020-000857

CRW and $\mathrm{NI}$ are joint first authors.

Received 9 December 2020 Revised 8 January 2021 Accepted 9 January 2021
Check for updates

(c) Author(s) (or their employer(s)) 2021. Re-use permitted under CC BY. Published by BMJ.

${ }^{1}$ Primary Care Research Centre, University of Southampton, Southampton, UK

${ }^{2}$ Clinical Trial Service Unit and Epidemiological Studies Unit (CTSU), Nuffield Department of Population Health, University of Oxford, Oxford, UK

Correspondence to Dr Christopher R Wilcox; christopher.wilcox@doctors. org.uk

\section{ABSTRACT}

Introduction Recent evidence suggests that influenza vaccination may offer protection against COVID-19 severity. Our aim was to quantify the association between influenza vaccination status and risk of hospitalisation or all-cause mortality in people diagnosed with COVID-19.

Methods A retrospective cohort study using routinely collected health records from patients registered to a General Practitioner (GP) practice in South West England within the Electronic Care and Health Information Analytics database. The cohort included 6921 people with COVID-19 during the first wave of the pandemic (1 January-31 July 2020). Data on influenza vaccination, hospitalisation and all-cause mortality were ascertained through linked clinical and demographic records. We applied propensity score methods (stabilised inverse probability of treatment weight) to quantify the association between influenza vaccination status and COVID-19 outcomes (hospitalisation or all-cause mortality).

Results 2613 (38\%) participants received an influenza vaccination between 1 January 2019 and COVID-19 diagnosis. Receipt of influenza vaccination was associated with a significantly lower odds of hospitalisation or allcause mortality (adjusted OR: $0.85,95 \% \mathrm{Cl} 0.75$ to 0.97 , $\mathrm{p}=0.02$ ), and $24 \%$ reduced odds of all-cause mortality (adjusted OR: $0.76,95 \% \mathrm{Cl} 0.64$ to 0.90 ).

Discussion Influenza vaccination was associated with a $15 \%-24 \%$ lower odds of severe COVID-19 outcomes. The current UK influenza vaccination programme needs urgent expansion as an integral component of the ongoing response plans to the COVID-19 pandemic.

\section{BACKGROUND}

COVID-19 caused by the SARS-CoV-2 is a rapidly evolving global public health emergency. Since the outbreak began, over 87 million cases have been reported globally, with over 1.8 million attributed deaths (as of 7 January 2020). ${ }^{1}$ Of those who develop symptoms, approximately $20 \%$ will have severe disease, including pneumonia and other lifethreatening complications. ${ }^{2}$ Older adults and those with underlying heath conditions are particularly susceptible to severe outcomes;

\section{Key messages}

We sought to determine the association between influenza vaccination status and risk of hospitalisation or all-cause mortality in people diagnosed with COVID-19.

- In people with COVID-19 during the first wave of the pandemic, we found that influenza vaccination was associated with a $15 \%-24 \%$ lower odds of hospitalisation or all-cause mortality.

- Influenza vaccination may have the potential to offer an additional means of mitigating serious adverse complications of COVID-19, and we therefore propose that a rapid expansion of the influenza vaccination programme is urgently needed as an integral component of the ongoing response to the COVID-19 pandemic.

however, recent evidence suggests that even mild COVID-19 may result in prolonged symptoms and/or long-term adverse health outcomes. ${ }^{3}$ The pandemic has carried a substantial economic burden globally, ${ }^{4}$ and several countries have experienced unprecedented rise in demand on healthcare systems far exceeding capacity to respond. ${ }^{5}$

There remain limited effective interventions to treat COVID-19. A number of vaccines against SARS-CoV-2 have recently been approved for use following promising clinical trials ${ }^{67}$; however, ongoing challenges remain regarding the manufacturing, distribution and accessibility of vaccines internationally, ${ }^{8}$ and there is an ongoing drive to identify whether any available treatments could be repurposed to prevent COVID-19 acquisition and/or disease severity. One area of interest is in the role of existing vaccines. Vaccination against specific pathogens with both live-attenuated and inactivated vaccines can induce adaptive and innate immunity in the form of neutralising antibodies and/or antigen-specific cellular immunity. Growing evidence demonstrates that vaccination 
may also have a role in wider protection against unrelated pathogens. ${ }^{910}$ The underlying mechanism for this phenomenon remains poorly understood and may occur by induction of innate immune responses, so-called trained innate immunity. ${ }^{911-14}$ It is hypothesised that influenza vaccination may potentially have a direct protective effect against COVID-19. ${ }^{69-17}$ This is in addition to its potential indirect benefits, such as reducing the burden of additional respiratory illness among both individual patients and healthcare services. ${ }^{18-20}$

This hypothesis has been supported by a limited number of observational studies, including five retrospective cohort studies ${ }^{151721-23}$ (three of which were limited by the use of aggregated data), demonstrating a negative association between influenza vaccination and COVID-19 outcomes including COVID-19 acquisition, severity and/ or mortality. One prospective study using individual-level data demonstrated a negative association between influenza vaccination and COVID-19 acquisition but did not report on subsequent COVID-19 outcomes. ${ }^{24}$

Further validation is required from large UK-based cohorts using individual-level data, exploring important outcomes including all-cause mortality and hospitalisation. This may help us better understand the impact of influenza vaccination in COVID-19 disease and inform the current influenza vaccination programme during the second wave of the pandemic. The aim of this study was to quantify the association between influenza vaccination status and COVID-19 outcomes including hospitalisation and all-cause mortality.

\section{METHODS}

\section{Study design}

Retrospective cohort analysis.

\section{Data source}

Data were obtained from the Care and Health Information Analytics (CHIA) database. This is an anonymous National Health Service (NHS) database that includes individual-level data from 1.5 million patients across 160 GP practices in Southern England. Data have been extracted from routine general practice records linked to local secondary care hospitals, including demographic data, primary/secondary care consultations, diagnoses, medications and outcomes of primary/secondary care investigations.

\section{Study population}

A cohort of individuals within the CHIA database diagnosed with COVID-19 during the first wave of the COVID-19 pandemic (1 January-31 July 2020) were identified. We included both confirmed and suspected cases. Confirmed cases had a positive RT-PCR assay for SARS-CoV-2 on nasal/pharyngeal swab. Clinically suspected cases were defined in accordance with Public Health England (PHE) guidance at the time of the first wave of the pandemic in the UK, when testing was not readily available. Further detail on the COVID-19 case definition has been reported in other databases, ${ }^{25}$ and our rationale for case definition can also be found in a consensus statement by the COVID-19 Primary Care Database Consortium. ${ }^{26}$ Recent evidence demonstrates that clinical suspicion of COVID-19 among GPs closely matches with confirmed cases and subsequent clinical outcomes. ${ }^{27}$

\section{Sociodemographic and clinical variables}

Data for the identified cohort were extracted from the CHIA database including age, sex, ethnicity, Index of Multiple Deprivation (IMD), electronic frailty index, comorbidities, smoking status, body mass index (BMI), medications and influenza vaccination status.

\section{Exposure}

Receipt of recent influenza vaccination was defined as an electronic recording of vaccination in the CHIA database between 1 January 2019 and the diagnosis of COVID-19.

\section{Outcomes}

Since death is a competing risk for hospitalisation, ${ }^{28}$ our primary outcome was a composite of hospitalisation or all-cause mortality. Our secondary outcome was allcause mortality alone. Incidence of hospitalisation and all-cause mortality was identified using linked hospital records and the Office for National Statistics. We did not analyse COVID-19 specific mortality (death within 28 days of diagnosis), as the exact dates of diagnosis were not sufficiently robust, and there was uncertainty about accuracy of electronic coding and certification of death nationally with regards to COVID-19 specific mortality in the first few months of the pandemic.

\section{Statistical analysis}

Baseline demographics/clinical characteristics of the study cohort were summarised. Categorical variables were summarised as frequencies and percentages, and continuous variables were as means and SD.

Since our primary exposure variable, influenza vaccination, is based on observational data (as opposed to allocation by randomisation), the group of patients that received the vaccine is not directly comparable with the group that did not receive the vaccine. The reasons why some people received the vaccination leads to so-called treatment indication bias. We attempted to address this confounding by treatment indication bias within marginal structural regression framework, because conventional regression adjustment may not be sufficient to address this issue. ${ }^{28}{ }^{29}$ Specifically, we estimated the comparative effectiveness of influenza vaccination by applying stabilised inverse probability of treatment weights (IPTW) for the receipt of influenza vaccination. ${ }^{30}$ Propensity score was estimated by fitting logistic regression using an a 
priori list of variables including age, sex, BMI, socioeconomic status (IMD), smoking status, frailty score (electronic frailty index), pre-existing comorbidities (chronic obstructive pulmonary disease, stroke, cancer, depression, peripheral arterial disease, rheumatoid arthritis, atrial fibrillation, dementia, chronic kidney disease, heart failure, learning disability, hypertension, other mental health disorder, cardiovascular disease, epilepsy, asthma, osteoporosis, coronary artery disease, osteoarthritis and diabetes) and the number of prescribed medications. The balance of the propensity score between the two vaccination groups was visually checked using histograms. The numerator for the stabilised IPTW was the marginal probability of vaccination receipt, estimated by fitting intercept-only logistic regression, for the group that received the influenza vaccine. The complement of this probability was the numerator for the group that did not receive the vaccination. Similarly, the propensity score was the denominator for the vaccinated group, while the complement of the propensity score was the denominator for the unvaccinated group. ${ }^{31}$ The effect estimates were reported as adjusted ORs and corresponding 95\% CIs. Statistical analyses were undertaken using Stata SE V.15.1 (StataCorp, College Station, Texas, USA).

\section{Sensitivity analysis}

To check the robustness of our analysis using stabilised IPTW, we estimated the pooled OR by stratifying the propensity score into deciles (10 equal groups). MantelHaenszel weighted pooled OR was estimated using Woolf approximation for the calculation of SE. ${ }^{32}$ In further sensitivity analysis, we defined influenza receipt only when it was administered within 6 months of COVID-19 pandemic in the UK (ie, when the vaccination was administered on or after 1 September 2019). Finally, we also performed an additional time-to-event analysis adjusting for month of COVID-19 diagnosis.

\section{Missing data}

There was missing data on ethnicity, BMI, smoking, IMD and age. Ethnicity is frequently missing from routinely collected primary care records. We removed this variable from the analyses for multiple reasons: (1) high amount of missing data on ethnicity (32.8\%), (2) very low number of outcome events in some subgroups (eg, there was one case of all-cause mortality in the Asian ethnic group) and (3) inadequate reduction of bias by traditional missing data imputation methods ${ }^{32} 33$ and (4) this geographical area of the UK has low proportion of ethnic minorities. For other variables with missing data, we used multiple imputation by chained equations assuming missing data were missing at random. We produced five sets of imputed datasets with 100 iterations in the burn-in period. ${ }^{3435}$ The non-missing variables used to impute the missing variables were sex, frailty score and pre-existing comorbidities.

\section{Ethical considerations}

CHIA is an anonymous NHS database, and all individuals have consented for collection of their medical records for inclusion in the database. Findings are reported as per Strengthening the Reporting of Observational Studies in Epidemiology and RECORD guidelines for observational studies of routinely collected data.

\section{Data availability}

Anonymised individual level data used in this study was extracted from the CHIA database. Direct requests for data sharing can be made to CHIA.

\section{Patient and public involvement}

Due to the nature of the study, patients or the public were not involved in the design, conduct, reporting or dissemination of the study

\section{RESULTS}

\section{Baseline characteristics}

A total of 6921 participants were included in the study, of which $774(11.2 \%)$ received a confirmed diagnosis of COVID-19 during the follow-up period, and 6147 (88.8\%) were diagnosed clinically. The mean age was 52.4 years $( \pm 24.5)$ and the majority of participants $(59.5 \%)$ were female. Most participants were white $(60.2 \%)$; however, ethnicity was not recorded in one-third of cases $(32.8 \%)$. Approximately half of participants were from the two least deprived socioeconomic status groups $(32.8 \%$ and $22.9 \%$ from IMD groups 5 and 4, respectively). Baseline characteristics of the study cohort are summarised in table 1 .

A total of 2613 (38\%) participants in the cohort had received influenza vaccination between 1 January 2019 and COVID-19 diagnosis (see table 1). Compared with the unvaccinated people, those who received influenza vaccination on average were older (mean 61.7 years vs 46.7 years) and frailer (mean frailty score 0.3 vs 0.1 ).

\section{Influenza vaccination and outcomes}

Baselines characteristics of the study cohort, stratified by incidence of the primary study outcome (hospitalisation or all-cause mortality), are displayed in table 2. Of the 2613 people who received an influenza vaccination, $372(14.2 \%)$ died during the follow-up time and 1166 $(44.6 \%)$ either died or were hospitalised. These proportions were $12.8 \%(\mathrm{n}=553)$ and $36.8 \%(\mathrm{n}=1584)$ in the 3755 people without vaccination. Those who died or had a hospital admission were on average older (mean 62.7 years vs 45.5 ), frailer (frailty score 0.2 vs 0.1 ) and a lower proportion were reported as white ethnicity $(52.0 \%$ vs $65.6 \%)$, with more having missing ethnicity data $(42.7 \%$ vs $26.3 \%$ ).

The overall distribution of the propensity score by influenza vaccination status is shown in figure 1 while the mean (SD) of the propensity score by deciles of 
Table 1 Baselines characteristics of people with COVID-19, within the CHIA database cohort, stratified by influenza vaccination status

\begin{tabular}{|c|c|c|c|}
\hline \multirow[b]{2}{*}{ Characteristics } & \multicolumn{3}{|c|}{ Influenza vaccination received } \\
\hline & Total & Yes & No \\
\hline $\mathrm{N}$ & 6921 & 2613 & 4308 \\
\hline Age baseline, mean (SD) & $52.4(24.5)$ & $61.7(24.0)$ & $46.7(22.9)$ \\
\hline Female, n (\%) & $4120(59.5)$ & $1577(60.4)$ & $2543(59.0)$ \\
\hline \multicolumn{4}{|l|}{ Ethnicity, n (\%) } \\
\hline Asian & $328(4.7)$ & $98(3.8)$ & $230(5.3)$ \\
\hline Black & $54(0.8)$ & $17(0.7)$ & $37(0.9)$ \\
\hline Missing data & $2273(32.8)$ & $704(26.9)$ & $1569(36.4)$ \\
\hline Mixed or other & $101(1.5)$ & $24(0.9)$ & $77(1.8)$ \\
\hline White & $4165(60.2)$ & $1770(67.7)$ & 2395 (55.6) \\
\hline \multicolumn{4}{|l|}{ Socioeconomic status (IMD), n (\%) } \\
\hline 1 (most deprived) & $617(8.9)$ & $244(9.3)$ & $373(8.7)$ \\
\hline 2 & $1055(15.2)$ & $328(12.6)$ & 727 (16.9) \\
\hline 3 & $1207(17.4)$ & 477 (18.3) & 730 (16.9) \\
\hline 4 & 1586 (22.9) & $599(22.9)$ & 987 (22.9) \\
\hline 5 (least deprived) & $2268(32.8)$ & $917(35.1)$ & $1351(31.4)$ \\
\hline Missing & $188(2.7)$ & $48(1.8)$ & $140(3.2)$ \\
\hline Frailty score, mean (SD) & $0.2(0.2)$ & $0.3(0.2)$ & $0.1(0.1)$ \\
\hline Frailty score, median (IQR) & $0.1(0.1-0.3)$ & $0.2(0.1-0.3)$ & $0.1(0.0-0.2)$ \\
\hline \multicolumn{4}{|l|}{ Comorbidities, n (\%) } \\
\hline Hypertension & 1396 (20.2) & $864(33.1)$ & $532(12.3)$ \\
\hline Stroke & $296(4.3)$ & $206(7.9)$ & $90(2.1)$ \\
\hline Asthma & 966 (14.0) & $576(22.0)$ & $390(9.1)$ \\
\hline Chronic obstructive pulmonary disease & $334(4.8)$ & $246(9.4)$ & $88(2.0)$ \\
\hline Coronary heart disease & $368(5.3)$ & $277(10.6)$ & $91(2.1)$ \\
\hline Heart failure & $196(2.8)$ & $144(5.5)$ & $52(1.2)$ \\
\hline Type 1 diabetes & $42(0.6)$ & $34(1.3)$ & $8(0.2)$ \\
\hline Type 2 diabetes & $559(8.1)$ & $397(15.2)$ & $162(3.8)$ \\
\hline CKD stage $3-5$ & $351(5.1)$ & $255(9.8)$ & $96(2.2)$ \\
\hline \multicolumn{4}{|l|}{ Smoking status, $\mathrm{n}(\%)$} \\
\hline Current smoker & $784(11.3)$ & $221(8.5)$ & $563(13.1)$ \\
\hline Ex-smoker & $2171(31.4)$ & 974 (37.3) & $1197(27.8)$ \\
\hline Never smoked & $3279(47.4)$ & $1215(46.5)$ & $2064(47.9)$ \\
\hline Missing data & $687(9.9)$ & $203(7.8)$ & $484(11.2)$ \\
\hline Body mass index & $27.9(6.9)$ & $28.7(7.4)$ & $27.4(6.5)$ \\
\hline \multicolumn{4}{|l|}{ Medication, n (\%) } \\
\hline Antiplatelets & $893(12.9)$ & $543(20.8)$ & $350(8.1)$ \\
\hline Oral anticoagulants & $651(9.4)$ & $368(14.1)$ & $283(6.6)$ \\
\hline ACE inhibitors & $977(14.1)$ & $554(21.2)$ & $423(9.8)$ \\
\hline Angiotensin receptor blockers & $473(6.8)$ & $286(10.9)$ & $187(4.3)$ \\
\hline Diuretics & $1051(15.2)$ & $627(24.0)$ & $424(9.8)$ \\
\hline Calcium channel blockers & $957(13.8)$ & $567(21.7)$ & $390(9.1)$ \\
\hline Beta blockers & $1117(16.1)$ & $567(21.7)$ & $550(12.8)$ \\
\hline Alpha blockers & $141(2.0)$ & $95(3.6)$ & $46(1.1)$ \\
\hline
\end{tabular}


Table 1 Continued

\begin{tabular}{lccc}
\hline \multirow{2}{*}{ Characteristics } & \multicolumn{3}{l}{ Influenza vaccination received } \\
\cline { 2 - 4 } & Total & Yes & No \\
\hline Insulin & $270(3.9)$ & $177(6.8)$ & $93(2.2)$ \\
Diabetic oral agents & $611(8.8)$ & $398(15.2)$ & $213(4.9)$ \\
Lipid-lowering drugs including statins & $1550(22.4)$ & $959(36.7)$ & $591(13.7)$ \\
\hline
\end{tabular}

CHIA, Care and Health Information Analytics; CKD, chronic kidney disease; IMD, Index of Multiple Deprivation.

the propensity score is shown in online supplementary table S1. The distribution shows a fairly strong common support and balanced distribution in the two vaccination groups.

In the stabilised IPTW-weighted regression model, influenza vaccination was associated with $15 \%$ reduced odds of hospitalisation or all-cause mortality (adjusted OR: $0.85,95 \%$ CI 0.75 to $0.97 ; \mathrm{p}=0.02)$ and $24 \%$ reduced odds of all-cause mortality (adjusted OR: 0.76, 95\% CI 0.64 to 0.90 ) (see table 3 ). Furthermore, on performing additional time-to-event analysis, we found that the results were identical to our primary analysis when we additionally adjusted for the month of COVID-19 diagnosis.

In the sensitivity analysis using pooled OR after stratification by deciles of propensity score, the results were almost identical for the primary outcome (adjusted OR: $0.87,95 \%$ CI 0.78 to $0.98, p=0.021$ ) with a slightly stronger effect for all-cause mortality (adjusted OR: 0.59, 95\% CI 0.51 to $0.69, \mathrm{p}<0.001$ ) (see table 3 ). In the sensitivity analysis with a modified definition of influenza vaccination (administered on or after 1 September 2020), the effects were almost identical to our primary analysis (adjusted OR: $0.85,95 \%$ CI 0.74 to $0.97, \mathrm{p}=0.016$ for hospitalisation or all-cause mortality, and $0.78,95 \%$ CI 0.66 to 0.92 , $\mathrm{p}=0.004$ for all-cause mortality).

\section{DISCUSSION}

Key findings

In this sample of 6921 participants who received a diagnosis of COVID-19 during the first wave of the pandemic (1 January-31 July 2019), we found that having prior influenza vaccination was associated with a 15\%-24\% lower odds of hospitalisation or all-cause mortality.

\section{Comparison with existing literature}

Our study is consistent with observational data from outside the UK demonstrating a negative association between influenza vaccination and COVID-19 acquisition, severity and mortality. ${ }^{12} 14183536$ An recent Italian retrospective study demonstrated a modest negative correlation between the percentage of adults aged $>65$ years who had received influenza vaccination and the percentage of COVID-19 deaths from each region of Italy (r: -0.59$).{ }^{15}$ Similarly, a US cohort of adults aged $\geq 65$ years demonstrated that a $10 \%$ increase in vaccination uptake was associated, on average, with a $28 \%$ decrease in the COVID-19 mortality rate. ${ }^{22}$ Another study examined influenza vaccination data from 34 countries (using Organisation for Economic Cooperation and Development data) with COVID-19 mortality from worldometer data and reported a negative correlation between influenza vaccination status and COVID-19 mortality $\left(\mathrm{R}^{2} 0.338\right) .{ }^{17}$ These studies were limited by the use of aggregated data, however. Two retrospective studies using patient-level data include a large Italian cohort study in which receipt of influenza vaccination was associated with 11\% lower odds of COVID-19 diagnosis, and among a subgroup of those aged $\geq 65$ years vaccinated in the first half of the vaccination programme, a $44 \%$ and $30 \%$ lower odds of hospitalisation and mortality, respectively. Another large retrospective cohort study in Brazil of 53752 hospitalised patients with COVID-19 found that receipt of recent influenza vaccination was associated with $7 \%, 17 \%$ and $16 \%$ lower odds of intensive care admission, requiring invasive respiratory support and mortality, respectively. Finally, there is one prospective observational study in the USA that includes individual patient level data through a registry of 11672 people tested for COVID-19 (of whom, 7\% tested positive). ${ }^{24}$ It reports that influenza vaccination was associated with a lower risk of acquiring COVID-19 $(\mathrm{p}<0.001$, OR not reported) but does not report on subsequent COVID-19 outcomes, including mortality.

Possible explanations for these findings include a direct protective effect against COVID-19 from influenza vaccination. Growing evidence, particularly from work studying BCG and measles vaccination, ${ }^{9} 1112$ as well as more recently from influenza vaccination, ${ }^{13} 14$ demonstrates that vaccination may have a role in wider protection against unrelated pathogens, in addition to inducing adaptive/innate immunity against specific pathogens. The underlying mechanisms for these non-specific effects remain poorly understood. One suggested mechanism is the induction of innate immune responses (including monocytes and natural killer cells), so-called trained innate immunity, following live attenuated vaccination, in a way that is independent of memory $\mathrm{T}$ or B cells. ${ }^{911-14}$ This trained immunity may confer non-specific protection against different pathogens, inducing upregulation of pattern recognition receptors and the secretion of proinflammatory cytokines through epigenetic and metabolic reprogramming. ${ }^{11-14}$ Another proposed mechanism is a process called 'emergency 
Table 2 Baselines characteristics of people with COVID-19 within the CHIA database cohort, stratified by incidence of the study outcomes (hospitalisation or all-cause mortality)

\begin{tabular}{|c|c|c|}
\hline \multirow[b]{2}{*}{ Characteristics } & \multicolumn{2}{|c|}{ Hospitalisation or all-cause mortality } \\
\hline & Yes & No \\
\hline $\mathrm{N}$ & 2750 & 4171 \\
\hline Received seasonal influenza vaccination, $\mathrm{n}(\%)$ & $1166(42.4)$ & $1447(34.7)$ \\
\hline Age baseline; mean (SD) & $62.7(22.8)$ & $45.5(23.1)$ \\
\hline Female, n (\%) & $1540(56.0)$ & $2580(61.9)$ \\
\hline \multicolumn{3}{|l|}{ Ethnicity, n (\%) } \\
\hline Asian & $106(3.9)$ & $222(5.3)$ \\
\hline Black & $17(0.6)$ & $37(0.9)$ \\
\hline Missing data & $1174(42.7)$ & $1099(26.3)$ \\
\hline Mixed or other & $23(0.8)$ & $78(1.9)$ \\
\hline White & $1430(52.0)$ & $2735(65.6)$ \\
\hline \multicolumn{3}{|l|}{ Socioeconomic status (IMD), n (\%) } \\
\hline 1 (most deprived) & $280(10.2)$ & $337(8.1)$ \\
\hline 2 & $479(17.4)$ & $576(13.8)$ \\
\hline 3 & $539(19.6)$ & $668(16.0)$ \\
\hline 4 & $611(22.2)$ & $975(23.4)$ \\
\hline 5 (least deprived) & $789(28.7)$ & 1479 (35.5) \\
\hline Missing & $52(1.9)$ & $136(3.3)$ \\
\hline Frailty score, mean (SD) & $0.2(0.2)$ & $0.1(0.2)$ \\
\hline Frailty score, median (IQR) & $0.2(0.1-0.3)$ & $0.1(0.0-0.2)$ \\
\hline \multicolumn{3}{|l|}{ Comorbidities, n (\%) } \\
\hline Hypertension & $586(21.3)$ & $810(19.4)$ \\
\hline Stroke & $154(5.6)$ & $142(3.4)$ \\
\hline Asthma & $306(11.1)$ & $660(15.8)$ \\
\hline Chronic obstructive pulmonary disease & $157(5.7)$ & $177(4.2)$ \\
\hline Coronary heart disease & $176(6.4)$ & $192(4.6)$ \\
\hline Heart failure & $115(4.2)$ & $81(1.9)$ \\
\hline Type 1 diabetes & $15(0.5)$ & $27(0.6)$ \\
\hline Type 2 diabetes & $256(9.3)$ & $303(7.3)$ \\
\hline CKD stage $3-5$ & $173(6.3)$ & $178(4.3)$ \\
\hline \multicolumn{3}{|l|}{ Smoking status, n (\%) } \\
\hline Current smoker & $291(10.6)$ & $493(11.8)$ \\
\hline Ex-smoker & $1027(37.3)$ & $1144(27.4)$ \\
\hline Never smoked & $1292(47.0)$ & $1987(47.6)$ \\
\hline Missing data & $140(5.1)$ & $547(13.1)$ \\
\hline Body mass index & $28.0(7.0)$ & $27.8(6.8)$ \\
\hline \multicolumn{3}{|l|}{ Medication, n (\%) } \\
\hline Antiplatelets & $561(20.4)$ & $332(8.0)$ \\
\hline Oral anticoagulants & $437(15.9)$ & $214(5.1)$ \\
\hline ACE inhibitors & $539(19.6)$ & $438(10.5)$ \\
\hline ARB & $236(8.6)$ & $237(5.7)$ \\
\hline Diuretics & $681(24.8)$ & $370(8.9)$ \\
\hline Calcium channel blockers & 547 (19.9) & $410(9.8)$ \\
\hline Beta blockers & 656 (23.9) & $461(11.1)$ \\
\hline
\end{tabular}


Table 2 Continued

\begin{tabular}{lcc}
\hline & \multicolumn{2}{l}{ Hospitalisation or all-cause mortality } \\
\cline { 2 - 3 } Characteristics & Yes & No \\
\hline Alpha blockers & $87(3.2)$ & $54(1.3)$ \\
\hline Insulin & $179(6.5)$ & $91(2.2)$ \\
\hline Diabetic oral agents & $363(13.2)$ & $248(5.9)$ \\
\hline Lipid-lowering drugs including statins & $894(32.5)$ & $656(15.7)$ \\
\hline
\end{tabular}

CHIA, Care and Health Information Analytics; CKD, chronic kidney disease; IMD, Index of Multiple Deprivation.

granulopoiesis' ${ }^{37}$ This is based on the recent observation that following BCG vaccination in newborns; for example, there is induction of the growth factor granulocyte colony-stimulating factor which, in turn, leads to the rapid production of neutrophils, resulting in additional protection from subsequent infection. ${ }^{38}$ Finally, influenza vaccination may also offer additional indirect protection against COVID-19 by reducing the burden of additional respiratory illness among both individual patients and healthcare services. ${ }^{18-20}$

\section{Strengths and limitations}

To our knowledge, this is the first study to examine influenza vaccination in relation to all-cause mortality and hospitalisation based on individual-patient data linked to demographic and clinical records in the UK. We applied robust analytic techniques to reduce the treatment indication bias that is inherent to observational studies. However, this does not completely rule out the possibility of residual and unknown confounding. A strength of the study is that it was drawn from a large database of individuals 160 GP practices across South West England; however, the cohort does include low representation of ethnic minority groups, and a relatively large proportion were from high socioeconomic backgrounds. Another limitation is our inability to analyse the effects by ethnicity

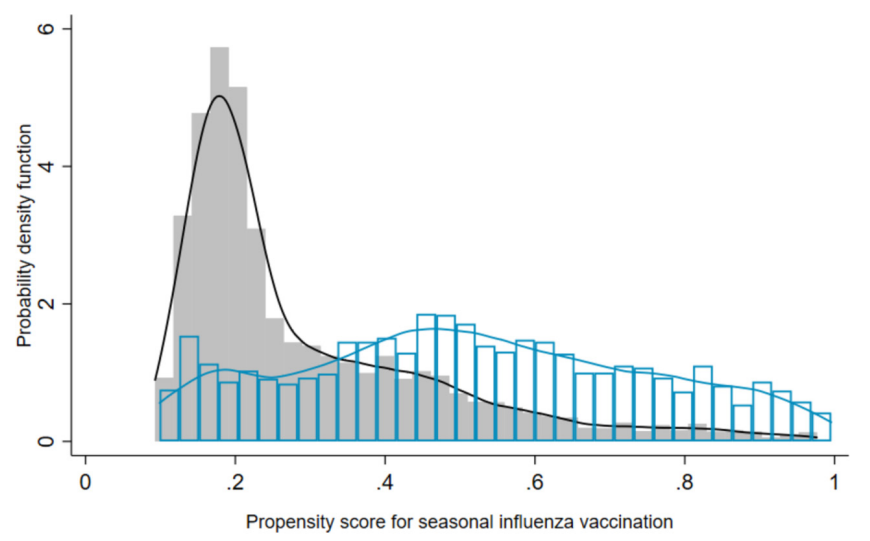

Not vaccinated Vaccinated

Figure 1 Distribution of the propensity score for patients who received seasonal influenza vaccination $(n=2613)$ compared with those who did not receive the vaccination $(\mathrm{n}=4308)$ since January 2019. due to large amount of missing data. Data being from a centralised single-payer healthcare system may limit the generalisability of these findings to other parts of the world.

The case definition of COVID-19 disease has also evolved over time, especially as testing has become more readily available in recent months. We included clinically suspected cases of COVID-19 alongside confirmed cases, in accordance with guidance from PHE during the first wave of the pandemic. Recent studies have shown that clinical suspicion of COVID-19 among GPs does closely match with confirmed cases and subsequent clinical outcomes, but it is still plausible that not all those with clinical cases had COVID-19. ${ }^{27}$ The clinical condition of patients at the time of diagnosis is also likely to have varied over the course of the study period (again as testing has become more readily available), as has the clinical management of patients, as clinicians have become more familiar with the COVID-19 over time. An additional time-to-event analysis in which we adjusted for the month of COVID-19 diagnosis showed identical results to our primary analysis, however. Our sample was also restricted to individuals with complete consultation notes over the study period. It is possible that individuals with incomplete records, mild symptoms only (or were asymptomatic) or attended hospital directly without first attending general practice may be under-represented in our sample. There is also a well-recognised 'frailty bias', ${ }^{39}$ whereby as people get older and frailer, vaccination rates tends to drop, and it is therefore plausible that this could have influenced our findings. However, we tried to take this into consideration by including frailty index, age and comorbidities within the regression model. Finally, the type of influenza vaccination given was not recorded in the database, and we are therefore unable to explore whether there is a difference between inactivated and live-attenuated vaccination on COVID-19 outcomes.

We used logistic regression for our primary analysis because the date of COVID-19 diagnosis, hospitalisation or deaths was not precise in the database. However, as stated above, additional time-to-event analysis adjusted for the month of COVID-19 diagnosis showed identical results. Furthermore, exact date of death was not always available for patients who died (only months and years of death were reported). Since the follow-up time was small, this is unlikely to have changed the estimates 
Table 3 Association between influenza vaccination and hospitalisation or all-cause mortality, and all-cause mortality alone, in people with COVID-19 within the CHIA database cohort $(n=6921)$

\begin{tabular}{|c|c|c|c|}
\hline Outcome & Model specification & OR $(95 \% \mathrm{Cl})$ & $P$ value \\
\hline \multirow[t]{3}{*}{ Hospitalisation or all-cause mortality } & Unadjusted & 1.39 (1.26 to 1.53$)$ & $<0.001$ \\
\hline & Stabilised IPTW* & $0.85(0.75$ to 0.97$)$ & 0.020 \\
\hline & Stratification and pooling & 0.87 (0.78 to 0.98$)$ & 0.021 \\
\hline \multirow[t]{3}{*}{ All-cause mortality } & Unadjusted & 1.13 (0.98 to 1.30$)$ & 0.097 \\
\hline & Stabilised IPTW† & 0.76 (0.64 to 0.90$)$ & 0.001 \\
\hline & Stratification and pooling & 0.59 (0.51 to 0.69$)$ & $<0.001$ \\
\hline
\end{tabular}

*IPTW: inverse probability of treatment weighting based on the propensity score for influenza vaccination stabilised by the marginal probability of vaccination in the study population.

$\dagger$ The propensity score was divided into deciles, and the OR for the association between influenza vaccination and the outcome was estimated in each of the deciles. Mantel-Haenszel-weighted pooled OR was estimated using Woolf approximation for the calculation of SE. $\mathrm{CHIA}$, Care and Health Information Analytics.

substantially. ${ }^{40}$ To verify this, we imputed the date as the 15th of the respective months and ran additionally sensitivity analyses on each of the outcomes using stabilised IPTW-weighted Cox's proportional hazards model to estimate the adjusted HR and 95\% CI. This robustly supported our findings; adjusted HR for the primary outcome (hospitalisation or all-cause mortality) and the secondary outcome (all-cause mortality) was $0.88(95 \%$ CI 0.79 to $0.98, \mathrm{p}=0.024)$ and 0.79 (95\% CI 0.66 to 0.94 , $\mathrm{p}=0.008)$, respectively.

\section{Implications of these findings}

Our results suggest that influenza vaccination may have the potential to offer an additional means of mitigating serious adverse complications of COVID-19. Further research should aim to validate these findings in larger more diverse cohorts. Additional work is also needed to examine differences between influenza vaccine subtypes and whether the duration from vaccination administration to COVID-19 acquisition is important.

\section{CONCLUSION}

In people with COVID-19 during the first wave of the pandemic, we found that influenza vaccination was associated with a $15 \%-24 \%$ lower odds of hospitalisation or all-cause mortality. An expansion of the influenza vaccination programme is needed as an integral component of the ongoing response to the COVID-19 pandemic.

Contributors CRW wrote the first draft of the manuscript, carried out data analysis and revised the manuscript. NI lead the data analysis and revised the manuscript. HD-M contributed to the inception of the study, designed the study methods, edited and contributed to subsequent versions of the manuscript. HDM is guarantor. All authors had access to, and verified, the underlying data.

Funding HD-M is a National Institute for Health Research (NIHR) funded Academic Clinical Lecturer at the University of Southampton and has received NIHR School of Primary Care Research (SPCR) funding to support her COVID-19 work (SPCR201410043). CW is an NIHR-funded Academic Clinical Fellow at the University of Southampton. Open access funding for publication of this article was provided by the University of Southampton. NI received salary support from the Nuffield Department of Population Health at the University of Oxford.
Disclaimer The views and opinions expressed by authors in this publication are those of the authors and do not necessarily reflect those of the UK NIHR or the Department of Health and Social Care.

Competing interests None declared.

Patient consent for publication Not required.

Ethics approval Ethical and governance approval for this specific study was obtained from the University of Southampton (ERGO 61740), and Care and Health Information Exchange Information Governance Group (CHIE IGG).

Provenance and peer review Not commissioned; externally peer reviewed.

Data availability statement Data are available on reasonable request. Anonymised individual level data used in this study was extracted from the Care and Health Information Analytics (CHIA) database. Direct requests for data sharing can be made to CHIA.

Supplemental material This content has been supplied by the author(s). It has not been vetted by BMJ Publishing Group Limited (BMJ) and may not have been peer-reviewed. Any opinions or recommendations discussed are solely those of the author(s) and are not endorsed by BMJ. BMJ disclaims all liability and responsibility arising from any reliance placed on the content. Where the content includes any translated material, BMJ does not warrant the accuracy and reliability of the translations (including but not limited to local regulations, clinical guidelines, terminology, drug names and drug dosages), and is not responsible for any error and/or omissions arising from translation and adaptation or otherwise.

Open access This is an open access article distributed in accordance with the Creative Commons Attribution 4.0 Unported (CC BY 4.0) license, which permits others to copy, redistribute, remix, transform and build upon this work for any purpose, provided the original work is properly cited, a link to the licence is given, and indication of whether changes were made. See: https://creativecommons.org/ licenses/by/4.0/.

\section{REFERENCES}

1 Worldometer. COVID-19 coronavirus pandemic, 2020.

2 Rubin D. Multiple imputation for nonresponse in surveys. New York, United States: John Wiley \& Sons Inc, 9AD.

3 Leung TYM, Chan AYL, Chan EW, et al. Short- and potential longterm adverse health outcomes of COVID-19: a rapid review. Emerg Microbes Infect 2020;9:2190-9.

4 Cutler DM, Summers LH. The COVID-19 Pandemic and the \$16 Trillion Virus. JAMA 2020;324:1495.

5 Verelst F, Kuylen E, Beutels P. Indications for healthcare surge capacity in European countries facing an exponential increase in coronavirus disease (COVID-19) cases, March 2020. Euro Surveill 2020;25.

6 Sultana J, Mazzaglia G, Luxi N. Potential effects of vaccinations on the prevention of COVID-19: rationale, clinical evidence, risks, and public health considerations. Expert Rev Vaccines 2020:1-18.

7 Ramasamy MN, Minassian AM, Ewer KJ, et al. Safety and immunogenicity of ChAdOx1 $\mathrm{nCoV}-19$ vaccine administered in a prime-boost regimen in young and old adults (COVO02): a 
single-blind, randomised, controlled, phase $2 / 3$ trial. The Lancet 2020;396:1979-93.

8 The Lancet. COVID-19 vaccines: no time for complacency. Lancet 2020;396:1607.

9 Chumakov K, Benn CS, Aaby P, et al. Can existing live vaccines prevent COVID-19? Science 2020;368:1187-8.

10 Higgins JPT, Soares-Weiser K, López-López JA, et al. Association of BCG, DTP, and measles containing vaccines with childhood mortality: systematic review. BMJ 2016;355:i5170.

11 Arts RJW, Moorlag SJCFM, Novakovic B, et al. Bcg vaccination protects against experimental viral infection in humans through the induction of cytokines associated with trained immunity. Cell Host Microbe 2018;23:89-100.

12 Covián C, Fernández-Fierro A, Retamal-Díaz A, et al. Bcg-Induced cross-protection and development of trained immunity: implication for vaccine design. Front Immunol 2019;10:2806.

13 Long BR, Michaelsson J, Loo CP, et al. Elevated frequency of gamma interferon-producing NK cells in healthy adults vaccinated against influenza virus. Clin Vaccine Immunol 2008;15:120-30.

14 Debisarun P, Struycken P J, et al. The effect of influenza vaccination on trained immunity: impact on COVID-19. MedRxiv 2020.

15 Marín-Hernández D, Schwartz R, Nixon D. Epidemiological evidence for association between higher influenza vaccine uptake in the elderly and lower COVID-19 deaths in Italy. J Med Virol 2020.

16 Lyu J, Miao T, Dong J, et al. Reflection on lower rates of COVID-19 in children: does childhood immunizations offer unexpected protection? Med Hypotheses 2020;143:109842.

17 Arokiaraj MC. Considering interim interventions to control COVID-19 associated morbidity and Mortality-Perspectives. Front. Public Health 2020;8:444.

18 Islam N, Khunti K, Majeed A. COVID-19, seasonal influenza and measles: potential triple burden and the role of flu and MMR vaccines. J R Soc Med 2020;113:485-6.

19 Richmond H, Rees N, McHale S, et al. Seasonal influenza vaccination during a pandemic. Hum Vaccin Immunother 2020:16:2219-21.

20 Maltezou HC, Theodoridou K, Poland G. Influenza immunization and COVID-19. Vaccine 2020;38:6078-9.

21 Ragni P, Marino M, Formisano D, et al. Association between exposure to influenza vaccination and COVID-19 diagnosis and outcomes. Vaccines 2020:8:675.

22 Zanettini C, Omar M, Dinalankara W, et al. Influenza vaccination and COVID19 mortality in the USA. medRxiv 2020 doi:10.1101/2020.06.24.20129817

23 Fink G, Orlova-Fink N, Schindler T, et al. Inactivated trivalent influenza vaccination is associated with lower mortality among patients with COVID-19 in Brazil. BMJ Evid Based Med 2020. doi:10.1136/bmjebm-2020-111549

24 Jehi L, Ji X, Milinovich A, et al. Individualizing risk prediction for positive coronavirus disease 2019 testing: results from 11,672 patients. Chest 2020;158:1364-75.
25 de Lusignan S, Lopez Bernal J, Zambon M, et al. Emergence of a novel coronavirus (COVID-19): protocol for extending surveillance used by the Royal College of general practitioners research and surveillance centre and public health England. JMIR Public Health Surveill 2020;6:e18606.

26 Dambha-Miller H, Griffin SJ, Young D. The use of primary care big data for COVID-19 research: a consensus statement from the COVID-19 primary care database Consortium. Annals of Family Medicine, COVID-19 Collection.

27 Joy M, Hobbs FDR, McGagh D, et al. Excess mortality from COVID-19 in an English sentinel network population. Lancet Infect Dis 2020;20.

28 Clift AK, Coupland CAC, Keogh RH, et al. Living risk prediction algorithm (QCOVID) for risk of hospital admission and mortality from coronavirus 19 in adults: national derivation and validation cohort study. BMJ 2020;371:m3731.

29 Bosco JLF, Silliman RA, Thwin SS, et al. A most stubborn bias: no adjustment method fully resolves confounding by indication in observational studies. J Clin Epidemiol 2010;63:64-74.

30 Robins JM, Hernán MA, Brumback B. Marginal structural models and causal inference in epidemiology. Epidemiology 2000;11:550-60.

31 Cole SR, Hernán MA, Cole H. Constructing inverse probability weights for marginal structural models. Am J Epidemiol 2008; $168: 656-64$

32 Desai RJ, Rothman KJ, Bateman BT, et al. A Propensity-scorebased fine stratification approach for confounding adjustment when exposure is infrequent. Epidemiology 2017;28:249-57.

33 Grundmeier RW, Song L, Ramos MJ, et al. Imputing missing Race/ Ethnicity in pediatric electronic health records: reducing bias with use of U.S. census location and Surname data. Health Serv Res 2015;50:946-60

34 Rubin D. Multiple imputation for nonresponse in surveys. New York, USA: John Wiley and Sons, 1987.

35 Azur MJ, Stuart EA, Frangakis C, et al. Multiple imputation by chained equations: what is it and how does it work? Int J Methods Psychiatr Res 2011;20:40-9.

36 Hughes RA, White IR, Seaman SR, et al. Joint modelling rationale for chained equations. BMC Med Res Methodol 2014:14:28.

37 Aaby P, Benn CS, Flanagan KL, et al. The non-specific and sexdifferential effects of vaccines. Nat Rev Immunol 2020;20:464-70.

38 Brook B, Harbeson DJ, Shannon CP, et al. Bcg vaccination-induced emergency granulopoiesis provides rapid protection from neonatal sepsis. Sci Transl Med 2020;12:eaax4517.

39 Andrew MK, Shinde V, Ye L, et al. The importance of frailty in the assessment of influenza vaccine effectiveness against Influenza-Related hospitalization in elderly people. J Infect Dis 2017;216:405-14.

40 Green MS, Symons MJ. A comparison of the logistic risk function and the proportional hazards model in prospective epidemiologic studies. J Chronic Dis 1983;36:715-23. 